\title{
IL-6 augments the invasiveness of U87MG human glioblastoma multiforme cells via up-regulation of MMP-2 and fascin-1
}

\author{
RONGHUI LI ${ }^{1}$, GANG LI $^{1}$, LIN DENG $^{1}$, QINGLIN LIU ${ }^{1}$, JUN DAI $^{2}$, JIE SHEN $^{1}$ and JIAN ZHANG ${ }^{2}$ \\ ${ }^{1}$ Department of Neurosurgery, Qilu Hospital, Shandong University; ${ }^{2}$ Institute of Immunopharmacology \\ and Immunotherapy, Shandong University School of Pharmaceutical Sciences, Jinan, P.R. China \\ Received January 5, 2010; Accepted February 12, 2010
}

DOI: 10.3892/or_00000795

\begin{abstract}
Invasion into adjacent brain parenchyma is the key cause of recurrent human glioblastoma multiforme (GBM). The role of interleukin 6 (IL-6) in the malignant progression of glioma remains undefined. Here, we found that IL-6 promotes the invasion of U87 MG human glioma cells but not in U343 cells. An advanced level of STAT3 activity, and increased expression and secretion of MMP-2, induced by IL-6 in U87 MG cells were observed. Blocking the STAT3 pathway with specific inhibitors of STAT3, JSI-124 or small interfering RNA (siRNA), inhibited the invasion of U87MG promoted by IL-6 with concomitant down-regulation of MMP-2. Furthermore, rapid up-regulation of fascin-1 (a cell motility-related protein) induced by IL-6 was apparent in U87MG cells. However, this up-regulation of fascin-1 was not inhibited by JSI-124 or siRNA. These results suggest that the STAT3 pathway and fascin-1 may play crucial roles in the invasiveness of U87MG cells promoted by IL-6.
\end{abstract}

\section{Introduction}

In the last fifty years, multimodality therapy has achieved only modest gains for malignant gliomas (1), which is one of the most common and fatal primary tumors in adults (2) and the second cause of cancer-related death in the young adults (3). It is generally believed that invasion and angiogenesis both play critical roles in the formation of recurrent tumors (4). It has been reported that blockade of VEGF (vascular endothelial growth factor)-mediated angiogenesis may promote invasive perivascular GBM (glioblastoma multiforme) growth $(5,6)$. Therefore, it is necessary to further characterize the mechanisms involved in invasion by GBM in order to discover new therapeutic targets.

High levels of circulating IL-6 have been observed in almost every type of tumor investigated and are associated

Correspondence to: Dr Gang Li, Department of Neurosurgery, Qilu Hospital, Shandong University, 107 Wenhua Xi Road, Jinan, Shandong 250012, P.R. China

E-mail: doctorligang@126.com

Key words: IL-6, invasiveness, glioma, STAT3, fascin-1, MMP-2 with poor prognosis (7). Recently, some studies have shown that IL-6 directly influences the invasiveness of colorectal, head and neck cancers $(8,9)$. In GBM, the autocrine regulation of IL-6 has been recognized since 1990 (10), and its aberrant expression and secretion have been found to be associated with GBM formation (11), angiogenesis, grade (12) and prognosis $(13,14)$. However, the role of IL-6 in the malignant progression of GBM remains unclear (7). In the present study, we demonstrated that IL-6 enhanced the invasive potential of GBM cells.

When the complex of IL- 6 and gp80 binds to gp130, gp130-associated janus kinases (JAKs) are activated. JAKs induce STAT3 phosphorylation and dimerization to promote its translocation to the nucleus where it regulates STAT3 transcriptional activation (15). IL-6 can also induce the expression and secretion of MMPs (matrix metalloproteinases) such as MMP-2 and MMP-9 in normal and tumor cells (16). In melanoma, phosphorylated STAT3 regulates the secretion of MMP-2 by directly binding to its promoter (17). Thus, we hypothesized that the STAT3 pathway was involved in the invasiveness of GBM promoted by IL-6.

Fascin-1 is a 55-kDa actin bindling protein which increases cell motility in multiple human malignancies $(18,19)$. In GBM, fascin-1 expression is correlated with histologic grade, prognosis (20), motility and invasiveness (21). In vitro invasiveness of U87 MG and U251 glioma cells was inhibited by fascin-1 siRNA treatment (21). Whether fascin-1 is involved in the invasion promoted by IL-6, and whether fascin-1 expression is regulated through the STAT3 pathway, were investigated in this study.

\section{Materials and methods}

Cell culture. The human malignant glioma cell lines, U87 MG and U343, were conserved in our laboratory. U343 cells were routinely cultured in RPMI-1640 (1640, Gibco) supplemented with $2 \mathrm{mM}$ L-glutamine and $10 \%$ fetal bovine serum (FBS, TBD, TianJin, China). U87 MG cells were cultured in Eagle's Minimum Essential Medium (EMEM, Gibco) adjusted to contain $1.5 \mathrm{~g} / 1$ sodium bicarbonate and supplemented with $2 \mathrm{mM}$ L-glutamine, $0.1 \mathrm{mM}$ non-essential amino acids, $1 \mathrm{mM}$ sodium pyruvate and $10 \% \mathrm{FBS}$. Cell cultures were maintained at $37^{\circ} \mathrm{C}$ in a humidified $5 \% \mathrm{CO}_{2}$ atmosphere. 
Antibodies and reagents. Anti-STAT3, anti-phospho-specific STAT3 (Tyr705, Ser727), anti-ß-actin antibodies and horseradish peroxidase-conjugated secondary antibody were obtained from Cell Signaling Technology. Anti-fascin-1 antibody was from Santa Cruz Biotechnology Inc. (Santa Cruz). Recombinant human IL-6 was purchased from Peprotech Inc. (Rochy Hill) and dissolved in serum-free medium prior to use. JSI-124 was from Calbiochem (San Diego) and dissolved in dimethyl sulfoxide (DMSO, Sigma). Immediately prior to use, a $9.72-\mathrm{mM}$ stock solution of JSI- 124 stored at $-20^{\circ} \mathrm{C}$ was diluted to the desired concentration with culture medium. The final concentration of DMSO for all treatments was maintained at $0.1 \%$.

Cell proliferation assay. Exponentially growing GBM cells were seeded into 96-well plates $\left(8 \times 10^{3}\right.$ cells/well, $\left.100 \mu \mathrm{l}\right)$ in serum-free medium and cultured for $6 \mathrm{~h}$, following which the medium was replaced with fresh serum-free medium containing $0-100 \mathrm{ng} / \mathrm{ml}$ of IL-6. After incubation for $12-36 \mathrm{~h}$, the Cell Counting Kit-8 (CCK-8, Dojindo) was used according to the manufacturer's instructions. The plates were read at A450 nm-630 nm using a microplate reader (Bio-Rad). The relative growth rate was calculated as follows: (experimental absorbance/untreated control absorbance-1) at each time-point.

Matrigel invasion assays. Invasion in vitro was measured in Transwell cell culture chambers (Costar, Corning). Matrigel (BD) coated filters with a pore size of $8 \mu \mathrm{m}$ were used. Cells that had been starved for $6 \mathrm{~h}$ were resuspended in serum-free culture medium containing IL-6 (0-100 ng/ml) and/or JSI-124 $(0-100 \mathrm{nM})$ and placed in the top chamber of the inserts $\left(3 \times 10^{4}\right.$ cells/well). Following incubation for $16 \mathrm{~h}$, the inserts were removed, the cells were fixed and stained with $0.1 \%$ crystal violet in methanol, viewed and counted under a light microscope. Photographs of representative microscopic fields were taken. Quantification of cell invasion was expressed as the mean count of stained cells in five random fields of each filter.

Small interfering RNA preparation and transfections. Small interfering RNA (siRNA) duplexes specific for STAT3 were designed according to Elbashir et al (22).The STAT3 target sequence was AGTCAGGTTGCTGGTCAAA and the siRNA was purchased from GenePharma (Shanghai, China). Nontargeting siRNA, was designed (GenePharma, CO) as a control for off-target effects caused by RNA interference. Transient transfections of siRNA (50 nM final concentration) against STAT3 were carried out using Lipofectamine 2000 (Invitrogen, Carlsbad, CA). Cells were plated at $60 \%$ confluency in EMEM containing 10\% serum without antibiotics and transfections were carried out $24 \mathrm{~h}$ later. Lipofectamine 2000 was diluted in serum-free EMEM for $5 \mathrm{~min}$ and the siRNA was diluted in serum-free EMEM. The two mixtures were combined and incubated for $20 \mathrm{~min}$ at room temperature to enable transfection complex formation. The complexes were subsequently added to the cells. Transfections with Lipofectamine 2000 alone served as controls. The supernatants were replaced with complete media $6 \mathrm{~h}$ later, and the cells were assayed with or without IL-6 treatment at $24 \mathrm{~h}$ after transfection.
$R N A$ isolation and RT-PCR. After treatment with various concentrations of IL- 6 for $9 \mathrm{~h}$ following starvation for $6 \mathrm{~h}$, cells were harvested and total RNA was extracted using TRIzol (Invitrogen). cDNA was synthesized in a $25-\mu 1$ reaction containing $2 \mu \mathrm{g}$ of total RNA using M-MLV reverse transcriptase (Promega). PCR primers for the amplification of $\beta$-actin and MMP-2 were synthesized by Sangon (Shanghai, China). The primer sequences were, $\beta$-actin: forward, 5'-ATC ATG TTT GAG ACC TTC AAC A-3'; reverse, 5'-CAT CTC TTG CTC GAA GTC CA-3'; MMP-2: forward, 5'-GGC CCT GTC ACT CCT GAG AT-3'; reverse, 5'-GGC ATC CAG GTT ATC GGG GA-3'. Amplification was performed over 30 cycles: $94^{\circ} \mathrm{C} / 45 \mathrm{sec}$ (denaturation), $58^{\circ} \mathrm{C} / 45 \mathrm{sec}$ (annealing), and $72^{\circ} \mathrm{C} / 5 \mathrm{~min}$ (extension). The PCR products were analyzed by electrophoresis and photographed using AlphaEaseFC software (Version 4.0.0, Alpha Innotech Corp.).

Zymography. Exponentially growing U87 MG cells were seeded into 24 -well plates $\left(4 \times 10^{4}\right.$ cells/well) in serum-free medium and cultured for $6 \mathrm{~h}$, following which the medium was replaced with fresh serum-free medium containing various concentrations of IL-6 and/or JSI-124. Following incubation for $16 \mathrm{~h}$, the supernatants were collected. The activity of MMP-2 in the supernatants was analyzed by SDS-PAGE with gels containing $0.1 \%$ gelatin $(\mathrm{w} / \mathrm{v})$ and $10 \%$ polyacrylamide (w/v). Coomassie Brilliant Blue staining and subsequent destaining with acetic acid result in decreased staining at the level of the electrophoretic migration of MMP-2. Quantification was performed by AlphaEaseFC software.

Western blot analysis. Exponentially growing U87 MG cells were cultured in serum-free medium for $6 \mathrm{~h}$, following which the medium was replaced with fresh serum-free medium containing various concentrations of IL-6 and/or JSI-124. Following incubation for $16 \mathrm{~h}$, the cells were collected. The cells were lysed in ice-cold lysis buffer and lysates were centrifuged at $13000 \mathrm{rpm}$ for $15 \mathrm{~min}$ at $4^{\circ} \mathrm{C}$. The whole cell extracts (30 $\mu \mathrm{g} /$ lane) were separated on $12 \%$ SDS-PAGE gels and then transferred to PVDF membranes (Millipore). After blocking in Tris-buffered saline with 5\% (w/v) non-fat dry milk, the membranes were probed with primary antibody at $4^{\circ} \mathrm{C}$ overnight. The membranes were then washed 3 times with TBST and incubated with horseradish peroxidaseconjugated secondary antibody. The proteins were visualized by the enhanced chemiluminescence (ECL) system (Pierce, Rockford, IL) using X-ray film. The bands were examined by densitometry using AlphaEaseFC software, with normalization of each band to their corresponding loading control.

Statistical analysis. All numeric data are presented as the mean \pm SD for at least three individual experiments. Statistical significance was determined by Student's t-test and accepted at $\mathrm{p}<0.05$.

\section{Results}

IL-6 promotes the invasiveness of U87MG cell in a Matrigel assay. The influence of IL-6 on the invasiveness of GBM cells was analyzed. As shown in Fig. 1A, Matrigel invasion by U87 MG glioma cells was enhanced by IL- 6 treatment for 
$\mathbf{A}$
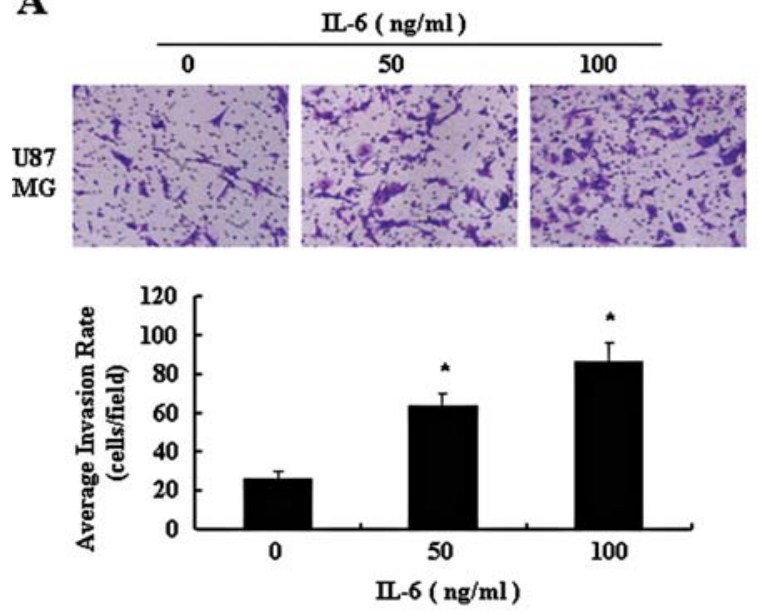

B

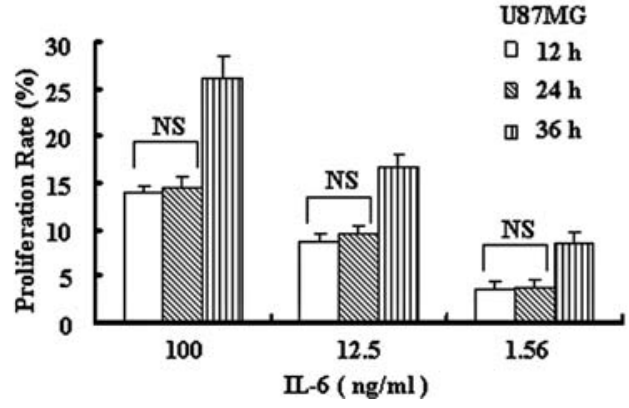

$\mathbf{C}$
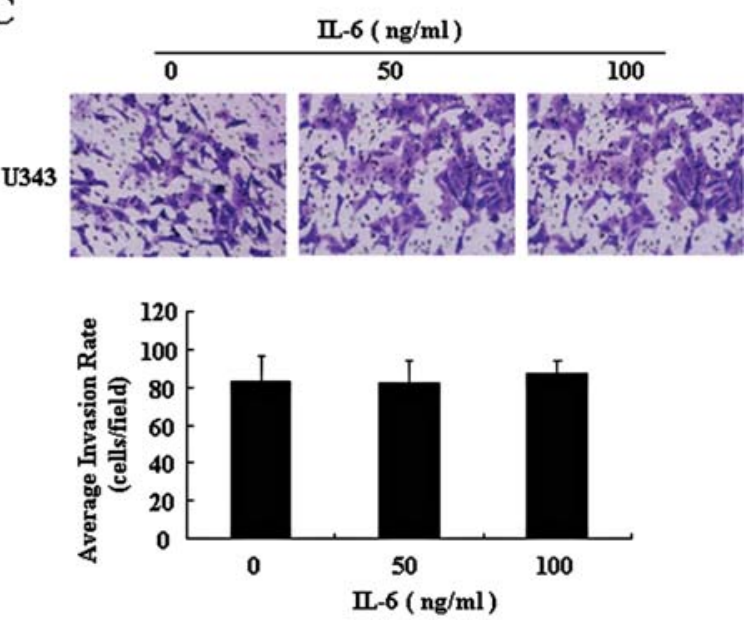

Figure 1. Promotion of Matrigel invasion by U87 MG cells treated with IL-6. (A) U87 MG cells were serum-starved for 6 h, resuspended in fresh serum-free culture medium containing the indicated concentrations of IL-6 (0-100 ng/ml) and placed in the upper chamber of the Transwell inserts $\left(3 \times 10^{4}\right.$ cells/well). After incubation for $16 \mathrm{~h}$, the inserts were removed and the cells were stained and counted. Average invasion rate was expressed as the mean count of stained cells in five random fields of each filter. (B) Exponentially growing GBM cells were seeded into 96-well plates (8x10 ${ }^{3}$ cells/well) in serum-free medium and cultured for $6 \mathrm{~h}$, following which the medium was replaced with fresh serum-free medium containing IL-6 (0-100 ng/ml). After incubation for 12-36 h, the CCK-8 assay was performed and absorbance was measured. The proliferation rate was calculated as follows: (experimental absorbance/untreated control absorbance-1) at each time-point. (C) U343 cells were treated in the same procedure like U87 MG cells as above. Bars represent mean \pm SE; NS, not significant; " $\mathrm{p}<0.05$. Original magnification $\mathrm{x} 200$.

$16 \mathrm{~h}$ in a dose-dependent manner. Since IL-6 was observed to promote the growth of U87MG cells (23), we used the CCK-8 assay to analyze whether the proliferation effect of IL-6 influenced the number of invasive cells. The results showed that at an IL- 6 dose range of $0-100 \mathrm{ng} / \mathrm{ml}$, there were no significant differences between the proliferation rates of cells treated for 12 or $24 \mathrm{~h}$ (Fig. 1B). However IL-6 did not show a promotion effect in U343 cells (Fig. 1C). These results suggest that IL-6 exerts different effects on the invasiveness in different glioma cell lines. In this study, we investigated the possible regulation pathway of the invasiveness in U87MG cells promoted by IL-6.

IL-6 up-regulates the expression levels of MMP-2 and fascin-1. Systemic metastases of malignant gliomas are seldom reported, but their infiltration into surrounding brain parenchyma is more frequently observed. Increased expression of MMPs is associated with the invasiveness of GBM, mainly due to proteolysis of extracellular matrix (ECM) components by MMPs (24). Fascin-1, the key filopodial bundling protein, is critical for enabling the cellular protrusions that mediate direct interactions with ECM and promote cell migration (21). Here, we analyzed whether the effect of IL-6 on the invasiveness of U87MG were associated with MMP-2 and fascin-1.

After treating cells with IL-6 for $9 \mathrm{~h}$, total RNA was extracted and RT-PCR was performed. As shown in Fig. 2A, the mRNA levels of MMP-2 were up-regulated in an IL-6 dose-dependent manner. According to gelatin zymography and Western blotting, IL-6 treatment for $16 \mathrm{~h}$ also increased MMP-2 production in the supernatant and the cells of U87MG cells in a dose-dependent manner (Fig. 2B and C). Western blotting was also performed to investigate the changes in fascin-1 expression in cells treated with IL- 6 for $15 \mathrm{~min}$ following serum starvation for $6 \mathrm{~h}$. As shown in Fig. 3A, the expression of fascin-1 increased rapidly in a dose-dependent manner, reaching the same levels after treatment with different concentrations of IL-6 for 16 h (Fig. 3B).

IL-6 enhances the phosphorylation of STAT3 in GMB cells. IL-6 signals primarily via the JAK/STAT3 pathway, and the STAT3 activity has been associated with the invasiveness of pancreatic cancer cells (25). Therefore, we investigated whether the up-regulation of MMP-2 and fascin-1 in invasive U87MG cells was due to STAT3 pathway activation by IL-6. Western blotting was used to analyze the effects of IL-6 on 
$\mathbf{A}$
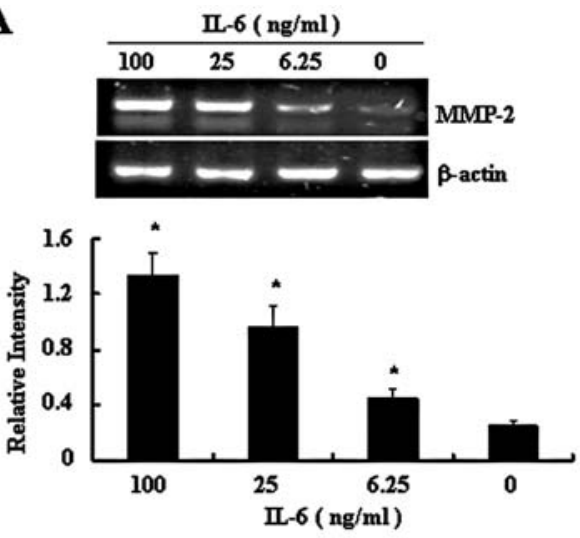

$\mathbf{C}$

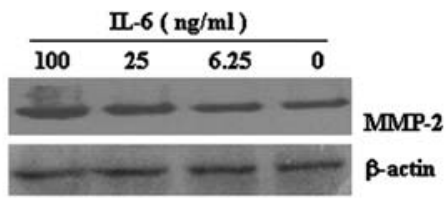

B
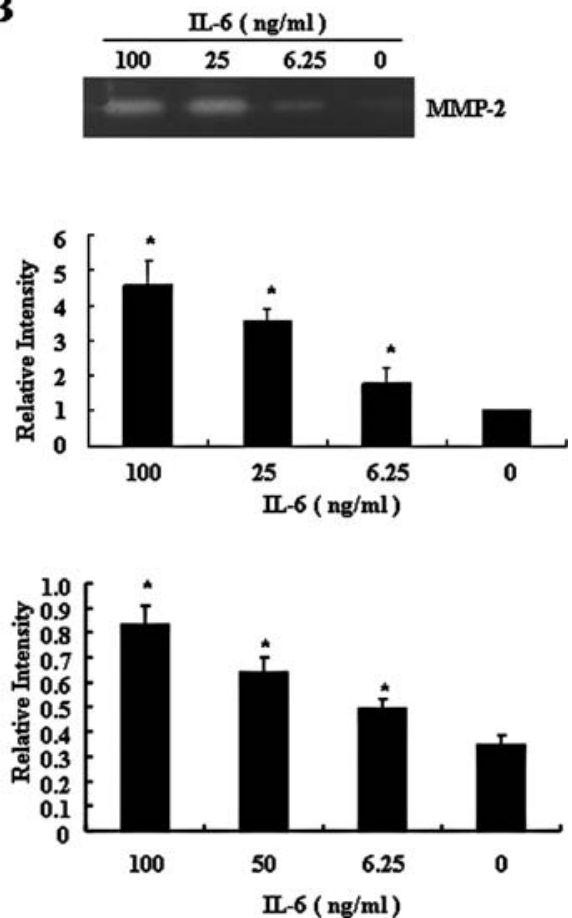

Figure 2. Up-regulation of MMP-2 expression in U87 MG cells treated with IL-6. After serum starvation for 6 h, cells were stimulated with various concentrations of IL-6 (0-100 ng/ml) in fresh serum-free medium. (A) After being stimulated for $9 \mathrm{~h}$, cells were harvested and total RNA was extracted. Semiquantitative RT-PCR was performed to determine MMP-2 mRNA expression. (B) After being stimulated for $16 \mathrm{~h}$, the conditioned media were collected and analyzed by gelatin zymography. (C) After being stimulated for $16 \mathrm{~h}$, the whole cell extracts were analyzed by Western blotting to examine MMP-2 expression. Levels of MMP-2 were expressed as relative fold changes in comparison to unstimulated samples. The histogram (A) represents the relative expression levels of each gene or protein after normalization to its corresponding internal control. Bars represent mean $\pm \mathrm{SE},{ }^{*} \mathrm{p}<0.05$.

$\mathbf{A}$
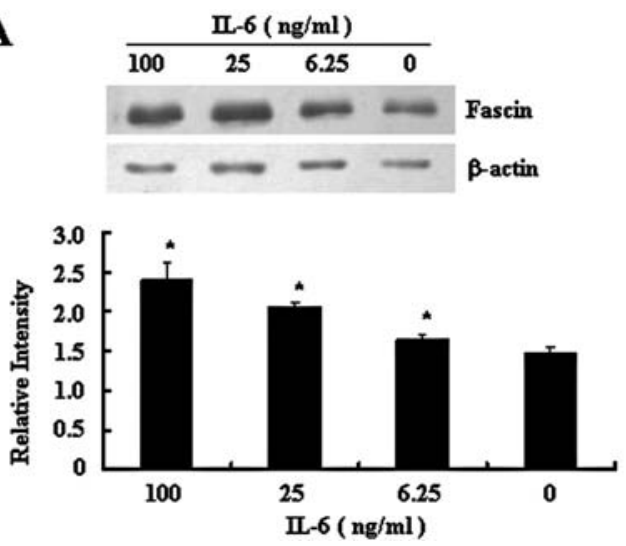

B
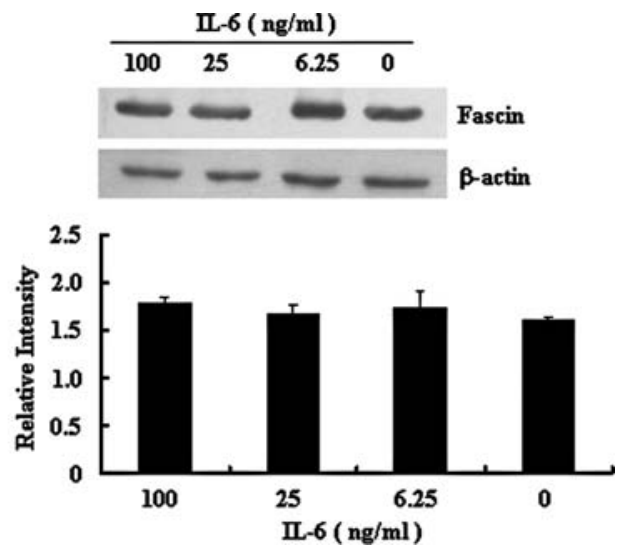

Figure 3. Changes of fascin-1 expression in U87 MG cells treated with IL-6. After being stimulated for 15 min (A) or 16 h (B), the whole cell extracts were analyzed by Western blotting to examine fascin-1 expression. The histograms represent the relative expression levels of each protein after normalization to its corresponding internal control. Bars represent mean $\pm \mathrm{SE},{ }^{*} \mathrm{p}<0.05$.

pSTAT3 (Tyr705, Ser727) levels in U87 MG cells, and the results showed that IL-6 increased both Tyr-705-phosphoSTAT3 and Ser-727-phospho-STAT3 levels in a dosedependent manner after treatment for $16 \mathrm{~h}$ (Fig. 4A).

MMP-2, but not fascin-1, is regulated by the STAT3 signaling pathway. To determine whether induction of MMP-2 and fascin- 1 expression by IL-6 in U87MG cells was regulated by the STAT3 signaling pathway, two specific inhibitor of STAT3, JSI-124 and siRNA were used to suppress the phosphorylation of STAT3 in U87 MG cells. As shown in Fig. 4, the STAT3 siRNA depleted the total STAT3 significantly in U87 MG cells at $24 \mathrm{~h}$ after transfection (Fig. 4B), and the levels of phosphorylated-STAT3 (Tyr705, Ser727) up-regulated by IL-6 $(100 \mathrm{ng} / \mathrm{ml})$ were also effectively suppressed by JSI-124 (Fig. 4C).

Matrigel invasion assays with STAT3 siRNA demonstrated that the depletion of total STAT3 could abate the invasiveness promoted by IL-6 (Fig. 5A). Similarly, JSI-124 reduced the number of invasive cells stimulated with IL-6 
A
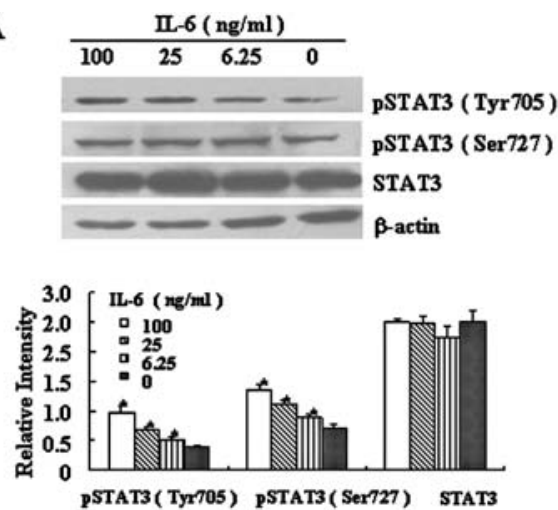

C

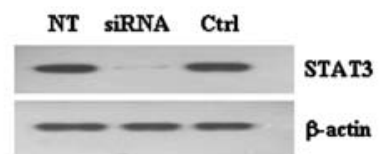

B
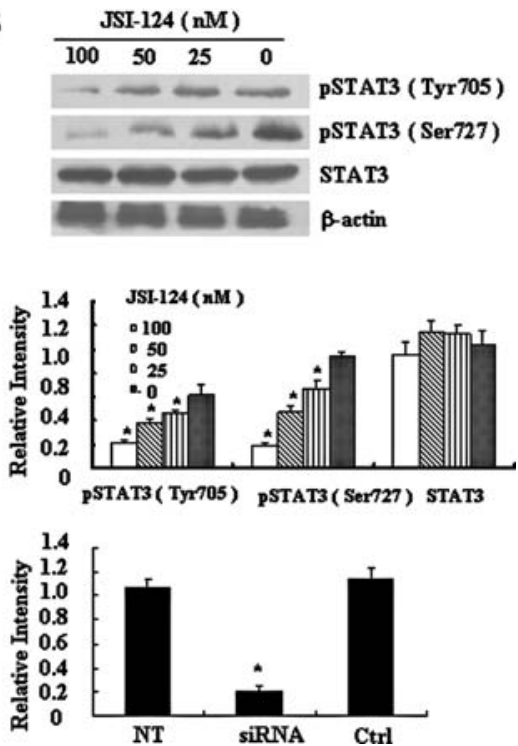

Figure 4. IL-6 enhances the phosphorylation of STAT3 in U87 MG cells and the enhancement can be inhibited by STAT3 siRNA or JSI-124. (A) After serum starvation for $6 \mathrm{~h}$, cells were stimulated with various concentrations of IL-6 (0-100 ng/ml) in fresh serum-free medium. After being stimulated for $16 \mathrm{~h}$, the whole cell extracts were analyzed by Western blotting to examine STAT3 expression. (B) After serum starvation for 6 h, cells were stimulated with various concentrations of JSI-124 (0-100 nM) and IL-6 (100 ng/ml) in fresh serum-free medium. After being stimulated for $16 \mathrm{~h}$, the whole cell extracts were analyzed by Western blotting to examine STAT3 expression. (C) At $24 \mathrm{~h}$ after STAT3 siRNA transfection, the whole cell extracts were analyzed by Western blotting to examine total STAT3 expression. Bars represent mean $\pm \mathrm{SE},{ }^{*} \mathrm{p}<0.05$. The histogram represents the relative expression levels of each protein after normalization to its corresponding internal control. NT, non-targeting siRNA transfected, siRNA, STAT3 siRNA transfected, Ctrl, Lipofectamine 2000 alone transfected.

A
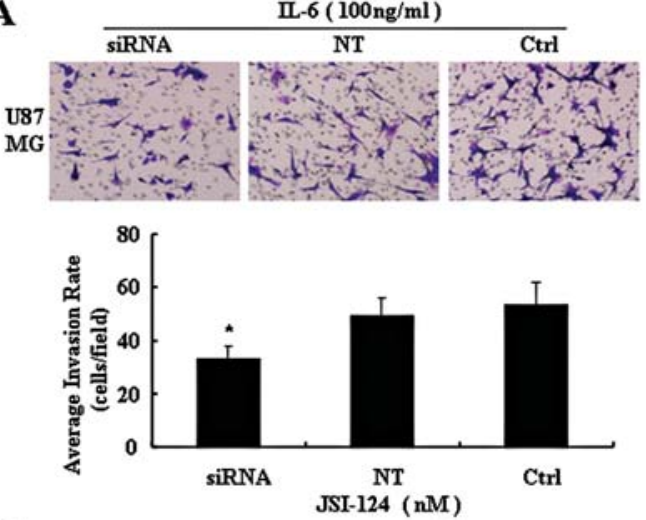

C

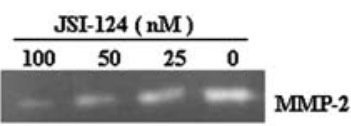

D
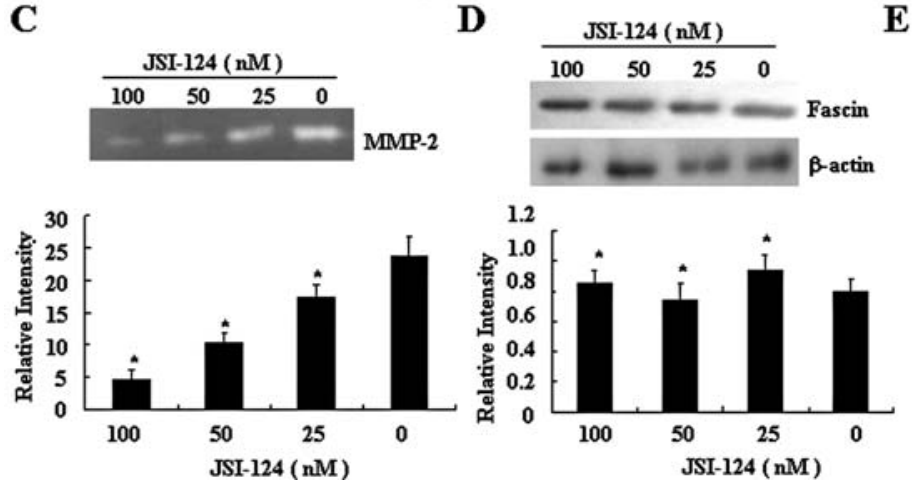

B
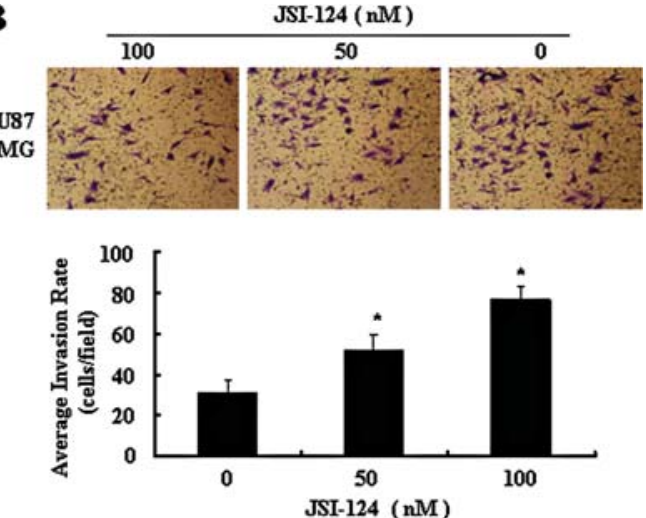

E
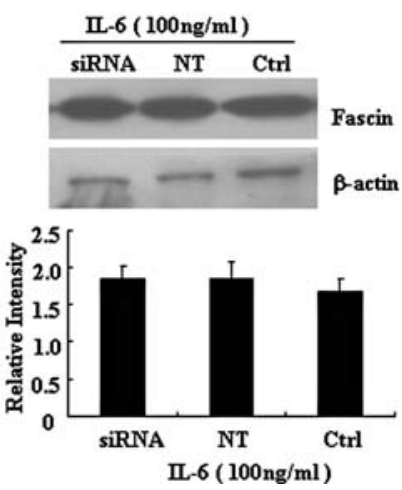

Figure 5. MMP-2, but not fascin-1, expression is regulated by the STAT3 signaling pathway in U87 MG cells. (A) IL-6 were added into the media (final concentration $100 \mathrm{ng} / \mathrm{ml}$ ) at $24 \mathrm{~h}$ after STAT3 siRNA transfection, and more $16 \mathrm{~h}$ later Matrigel invasion analysis was performed as described in Materials and methods. Average invasion rate is expressed as the mean count of stained cells in five random fields of each filter. (B-D) After the serum starvation for $6 \mathrm{~h}$, cells were treated with IL-6 $(100 \mathrm{ng} / \mathrm{ml})$ and various concentrations of JSI-124 (0-100 $\mathrm{nM})$ in fresh serum-free medium for $16 \mathrm{~h}$. Matrigel invasion analysis was performed as described above (B). The conditioned media were collected and analyzed by gelatin zymography. Levels of MMP-2 are expressed as relative fold changes in comparison to unstimulated samples (C). The whole cell extracts were analyzed by Western blotting to examine fascin-1 expression (D). (E) Since $24 \mathrm{~h}$ after STAT3 siRNA transfection, the cells were treated with IL-6 (100 ng/ml) for $15 \mathrm{~min}$. The whole cell extracts were analyzed by Western blotting to examine fascin-1 expression. The histograms represent the relative expression levels of each protein after normalization to its corresponding internal control. Bars represent mean $\pm \mathrm{SE},{ }^{*} \mathrm{p}<0.05$. Original magnification $\mathrm{x} 200$. NT, non-targeting siRNA transfected, siRNA, STAT3 siRNA transfected, Ctrl, Lipofectamine 2000 alone transfected. 
(100 ng/ml) in a dose-dependent manner as shown in Fig. 5B, while also suppressing the levels of active MMP-2 production induced by IL-6 (Fig. 5C).

However, JSI-124 (0-100 nM) did not inhibit the upregulation of fascin-1 expression activated by IL-6 (100 ng/ ml; Fig. 5D). Since the down-regulation of p-STAT3 (Tyr705, Ser727) by JSI-124 was a relatively slow event (26), we only investigated the fascin-1 level after 16-h treatment with IL-6 and JSI-124. But the fascin-1 expression was not yet reduced in the U87 MG cells transfected by STAT3 siRNA after being treated with IL-6 for 15 min (Fig. 5E).

\section{Discussion}

Many biological activities mediated by the cytokine IL-6 are well characterized. However, it remains unknown exactly how IL-6 contributes to the malignant progression of glioma, even though its autocrine regulation in GBM has been known for decades. Further study is still required to define the role of IL-6 in the malignant progression of glioma (7). In this study, we demonstrated that IL-6 promoted the invasion of Matrigel by human U87MG cells. However, this promotion is not observed in U343 cells. These results suggest that the invasion promotion of IL- 6 in GBM and its relative molecular pathways are cell line dependent.

JAK/STAT3 is the major signaling pathway triggered by IL-6. Aberrantly activated STAT3 has been found not only in GBM cells and animal tumor tissues (27), but also in human clinical GBM samples $(27,28)$. In our previous study, we found that inhibiting the STAT3 signaling pathway with JSI-124 could effectively promote apoptosis in, and inhibits the proliferation of, GBM cells (26). Our present study demonstrated that blocking the STAT3 pathway with JSI-124 or siRNA inhibited the invasiveness of U87MG cells promoted by IL-6, which suggested that STAT3 activation was required for this process. In the invasion promoted by IL-6, both the upregulation of MMP-2 expression and the activation of STAT3 were observed. When the activation of STAT3 induction was suppressed, the MMP-2 expression was downregulated, while the invasion induced by IL- 6 was also inhibited. It can be seen that, the up-regulation of MMP-2 through activation of STAT3 is one of the pathways in the invasiveness of U87 MG cells enhanced by IL-6.

Fascin-1, which plays an important role in the migration of GBM cells, increases the mechanical stiffness of filopodial protrusions by promoting actin-bundling. Data suggest that up-regulated fascin-1 is commonly correlated with the aggressive behavior of carcinoma cells (19). Hwang et al (21) have shown that fascin has an important role in glioma cell motility and invasiveness. In this study, a rapid dose-dependent increase in fascin-1 was observed after stimulation of U87 MG cells with IL-6 for $15 \mathrm{~min}$. Accordingly, we suggest that fascin-1 is involved in the invasion promoted by IL-6. Interestingly, the expression of fascin-1 reached the same maximal levels after treatment with different concentrations of IL- 6 for $16 \mathrm{~h}$, even without exogenous IL-6 treatment. We speculate that signaling via IL-6 may up-regulate the expression of fascin-1, and sustain the basic level of fascin- 1 in GBM cells. To explain why fascin-1 protein expression in U87 MG cells reached a high level after $16 \mathrm{~h}$ without exogenous IL-6 treatment, we hypothesize that IL-6 accumulated by autocrine regulation sustains the expression level of fascin-1 in U87 MG cells. In contrast, neither JSI-124 nor siRNA could decrease the levels of fascin-1 in U87 MG cells treated with IL-6 for $16 \mathrm{~h}$, although it did inhibit STAT3 activation. This suggested that the up-regulation of fascin-1 activated by IL- 6 was not mediated by the STAT3 pathway, which may also explain why inactivation or depletion of STAT3 did not completely abrogate the invasion promoted by IL-6. Further study is required to determine whether the molecular basis for the upregulation of fascin-1 expression is transcriptional or posttranscriptional.

In summary, IL-6 promotes the invasiveness of human U87MG cells by a process that requires STAT3 activity. Inhibition of STAT3 by JSI-124 suppressed the invasiveness of cells incubated with IL-6. Fascin-1 is involved in this invasive process but not regulated by STAT3. Given that IL-6 mediates VEGF up-regulation via a direct interaction between STAT3 and Sp1 in GBM cells (15), anti-IL-6 therapy promises to combine anti-angiogenic with anti-invasion approaches to glioma. Therefore, anti-IL-6 strategies targeting its downstream pathways and proteins may be worth exploring for the treatment of recurrent GBM.

\section{Acknowledgements}

This study was supported by Natural Science Foundation of China (No.30872645) and Natural Science Foundation of Shandong Province (No.2008C57).

\section{References}

1. Fomchenko EI and Holland EC: Mouse models of brain tumors and their applications in preclinical trials. Clin Cancer Res 12: 5288-5297, 2006.

2. Chen SH, Gillespie GY and Benveniste EN: Divergent effects of oncostatin $\mathrm{M}$ on astroglioma cells: influence on cell proliferation, invasion and expression of matrix metalloproteinases. Glia 53: 191-200, 2006.

3. Lonser RR, Walbridge S, Vortmeyer AO, et al: Induction of glioblastoma multiforme in non-human primates after therapeutic doses of fractionated whole-brain radiation therapy. J Neurosurg 97: 1378-1389, 2002.

4. Bello L, Giussani C, Carrabba G, et al: Suppression of malignant glioma recurrence in a newly developed animal model by endogenous inhibitors. Clin Cancer Res 8: 3539-3548, 2002.

5. Rubenstein JL, Kim J, Ozawa T, Zhang M, Westphal M, Deen DF and Shuman MA: Anti-VEGF antibody treatment of glioblastoma prolongs survival but results in increased vascular cooption. Neoplasia 2: 306-314, 2000.

6. Steeg PS: Angiogenesis inhibitors: motivators of metastasis? Nat Med 9: 822-823, 2003.

7. Hong DS, Angelo LS and Kurzrock R: Interleukin-6 and its receptor in cancer: implications for translational therapeutics. Cancer 110: 1911-1928, 2007.

8. Hsu CP and Chung YC: Influence of interleukin-6 on the invasiveness of human colorectal carcinoma. Anticancer Res 26: 4607-4614, 2006.

9. Kanazawa T, Nishino H, Hasegawa M, Ohta Y, Iino Y, Ichimura $\mathrm{K}$ and Noda Y: Interleukin-6 directly influences proliferation and invasion potential of head and neck cancer cells. Eur Arch Otorhinolaryngol 264: 815-821, 2007.

10. Van Meir E, Sawamura Y, Diserens AC, Hamou MF and De Tribolet N: Human glioblastoma cells release interleukin 6 in vivo and in vitro. Cancer Res 50: 6683-6688, 1990.

11. Weissenberger J, Loeffler S, Kappeler A, et al: IL-6 is required for glioma development in a mouse model. Oncogene 23: 3308-3316, 2004.

12. Rolhion C, Penault-Llorca F, Kemeny JL, et al: Interleukin-6 overexpression as a marker of malignancy in human gliomas. J Neurosurg 94: 97-101, 2001. 
13. Tchirkov A, Khalil T, Chautard E, et al: Interleukin-6 gene amplification and shortened survival in glioblastoma patients. Br J Cancer 96: 474-476, 2007.

14. Chang CY, Li MC, Liao SL, Huang YL, Shen CC and Pan HC: Prognostic and clinical implication of IL-6 expression in glioblastoma multiforme. J Clin Neurosci 12: 930-933, 2005.

15. Loeffler S, Fayard B, Weis J and Weissenberger J: Interleukin-6 induces transcriptional activation of vascular endothelial growth factor (VEGF) in astrocytes in vivo and regulates VEGF promoter activity in glioblastoma cells via direct interaction between stat 3 and sp1. Int J Cancer 115: 202-213, 2005.

16. Kossakowska AE, Edwards DR, Prusinkiewicz C, et al: Interleukin-6 regulation of matrix metalloproteinase (MMP-2 and MMP-9) and tissue inhibitor of metalloproteinase (TIMP-1) expression in malignant non-Hodgkin's lymphomas. Blood 94: 2080-2089, 1999.

17. Xie TX, Wei D, Liu M, et al: Stat3 activation regulates the expression of matrix metalloproteinase- 2 and tumor invasion and metastasis. Oncogene 23: 3550-3560, 2004.

18. Grothey A, Hashizume R, Ji H, et al: C-erbb-2/her-2 up-regulates fascin, an actin-bundling protein associated with cell motility, in human breast cancer cell lines. Oncogene 19: 4864-4875, 2000.

19. Hashimoto Y, Skacel M and Adams JC: Roles of fascin in human carcinoma motility and signaling: prospects for a novel biomarker? Int J Biochem Cell Biol 37: 1787-1804, 2005.

20. Gunal A, Onguru O, Safali M and Beyzadeoglu M: Fascin expression in glial tumors and its prognostic significance in glioblastomas. Neuropathology 28: 382-386, 2008.
21. Hwang JH, Smith CA, Salhia B and Rutka JT: The role of fascin in the migration and invasiveness of malignant glioma cells. Neoplasia 10: 149-159, 2008.

22. Elbashir SM, Harborth J, Lendeckel W, Yalcin A, Weber K and Tuschl T: Duplexes of 21-nucleotide RNAs mediate RNA interference in cultured mammalian cells. Nature 411: 494-498, 2001.

23. Goswami S, Gupta A and Sharma SK: Interleukin-6-mediated autocrine growth promotion in human glioblastoma multiforme cell line U87 MG. J Neurochem 71: 1837-1845, 1998.

24. Rao JS: Molecular mechanisms of glioma invasiveness: the role of proteases. Nature Rev 3: 489-501, 2003

25. Huang C, Cao J, Huang KJ, Zhang F, Jiang T, Zhu L and Qiu ZJ: Inhibition of STAT3 activity with AG490 decreases the invasion of human pancreatic cancer cells in vitro. Cancer Sci 97: 1417-1423, 2006

26. Su Y, Li G, Zhang X, Gu J, Zhang C, Tian Z and Zhang J: JSI-124 inhibits glioblastoma multiforme cell proliferation through $\mathrm{G}(2) / \mathrm{m}$ cell cycle arrest and apoptosis augment. Cancer Biol Ther 7: 1243-1249, 2008.

27. Iwamaru A, Szymanski S, Iwado E, et al: A novel inhibitor of the STAT3 pathway induces apoptosis in malignant glioma cells both in vitro and in vivo. Oncogene 26: 2435-2444, 2007.

28. Schaefer LK, Ren Z, Fuller GN and Schaefer TS: Constitutive activation of STAT3alpha in brain tumors: localization to tumor endothelial cells and activation by the endothelial tyrosine kinase receptor (VEGFR-2). Oncogene 21: 2058-2065, 2002. 